\title{
Reconstruction Filter Type
}

National Cancer Institute

\section{Source}

National Cancer Institute. Reconstruction Filter Type. NCI Thesaurus. Code C106564.

A classification of the reconstruction filter used to suppress noise, enhance edges,

perform resolution recovery and smooth an image. 